\title{
Classical transients and the support of open quantum maps
}

\author{
Gabriel G. Carlo, ${ }^{1, *}$ D. A. Wisniacki, ${ }^{2}$ Leonardo Ermann, ${ }^{1}$ R. M. Benito, ${ }^{3}$ and F. Borondo ${ }^{4}$ \\ ${ }^{1}$ Departamento de Física, Comisión Nacional de Energía Atómica, Avenida del Libertador 8250, 1429 Buenos Aires, Argentina \\ ${ }^{2}$ Departamento de Física and IFIBA, FCEyN, UBA Ciudad Universitaria, Pabellón 1, Ciudad Universitaria, 1428 Buenos Aires, Argentina \\ ${ }^{3}$ Grupo de Sistemas Complejos and Departamento de Física, Escuela Técnica Superior de Ingenieros Agrónomos, \\ Universidad Politécnica de Madrid, 28040 Madrid, Spain \\ ${ }^{4}$ Departamento de Química and Instituto de Ciencias Matemáticas (ICMAT), Universidad Autónoma de Madrid, \\ Cantoblanco, 28049 Madrid, Spain
}

(Received 24 July 2012; revised manuscript received 9 October 2012; published 17 January 2013)

\begin{abstract}
The basic ingredients in a semiclassical theory are the classical invariant objects serving as a support for quantization. Recent studies, mainly obtained on quantum maps, have led to the commonly accepted belief that the classical repeller - the set of nonescaping orbits in the future and past evolution-is the object that suitably plays this role in open scattering systems. In this paper we present numerical evidence warning that this may not always be the case. For this purpose we study recently introduced families of tribaker maps [L. Ermann, G. G. Carlo, J. M. Pedrosa, and M. Saraceno, Phys. Rev. E 85, 066204 (2012)], which share the same asymptotic properties but differ in their short-time behavior. We have found that although the eigenvalue distribution of the evolution operator of these maps follows the fractal Weyl law prediction, the theory of short periodic orbits for open maps fails to describe the resonance eigenfunctions of some of them. This is a strong indication that new elements must be included in the semiclassical description of open quantum systems. We provide an interpretation of the results in order to have hints about them.
\end{abstract}

DOI: 10.1103/PhysRevE.87.012909

PACS number(s): 05.45.Mt, 03.65.Sq

\section{INTRODUCTION}

The strong recent interest in the study of open quantum systems has demonstrated that this area is still in a very early stage of development, especially compared with the knowledge that we have of the closed counterparts. As a consequence, it has become an extremely active topic in fundamental physics [1]. The most important advance concerns scattering systems, where the fractal Weyl law (FWL) for the number of long-lived resonances has been conjectured and tested in different examples [2]. The corresponding classical invariant distribution, i.e., the fractal hyperbolic set of all trajectories nonescaping in the past and future (the repeller), plays a fundamental role in the interpretation of the quantum spectrum of quasibound (resonant) states. According to this law [3] the number of long-lived resonances scales with Planck constant as $\hbar^{-d / 2}$, where $d+1$ is the fractal dimension of the repeller. After the proof of the fractal Weyl upper bound for a Hamiltonian flow showing a fractal trapped set [4], results that support the validity of the conjectured law for both smooth and hard wall potentials [5] followed. However, open quantum maps provide the ideal arena for this kind of study, due to its computational simplicity [6], and accordingly, we focus on them in this work.

Another recent advance in the understanding of scattering systems has been the development of the short periodic orbit (PO) theory for open quantum maps [7]. This theory makes use of the shortest POs living in the repeller to construct a classically motivated basis in which the quantum nonunitary operators corresponding to the classical maps can be adequately expressed. It has been shown that there is a connection between the FWL and the possibility of obtaining

*carlo@tandar.cnea.gov.ar the quantum long-lived resonances by using the detailed classical information on the repeller, i.e., the embedded trajectories [8].

As a result of all this evidence, the repeller is commonly accepted to be the fundamental invariant classical structure in phase space able to explain the quantum mechanics of open systems. However, in a recent publication [9], it was shown for a set of maps sharing the same repeller and asymptotic features (including the escape rate), but having different classical transient behavior, that the quantum properties of the resonances can differ strongly from this view. This suggests that also transients may play a fundamental role in the description of open quantum systems.

In this paper we further investigate this issue, by presenting a twofold numerical calculation on the tribaker map families introduced in [9]. These families differ in the way in which the openings are defined. In the so-called shift family the area of the opening is the same for all of its members, while in the intersection family this area changes. First, we have studied the statistical behavior of the resonances of the quantum maps of the shift and intersection families. We have verified that the resonance distributions follow the FWL for these two kinds of maps. But the systematic imposed by the FWL start to work from a dimension of the Hilbert space that depends on the characteristics of the map. Second, we have tested the ability of the theory of short POs for open quantum maps to reproduce the eigenvalues and the corresponding resonance eigenfunctions. This approach is used as a tool to investigate the actual classical support of the quantum resonances, finding that the quantum information on some of the shift family members cannot be reproduced. These results cast doubts on the commonly accepted belief that the repeller is just enough to support the quantization of open maps. By interpreting the results we find some hints about the missing ingredients, diffraction effects due to the sharpness of opening borders 
not fully considered in the formulation being an undoubtedly central one.

The organization of the paper is as follows: In Sec. II we introduce the features of the shift and intersection families of open tribaker maps. In Sec. III we study the effects of classical transients on the spectral statistics. The same is done in Sec. IV regarding the classical support of open quantum maps by using the theory of short POs as the main tool. Finally, in Sec. V we present the final discussion and our perspectives for future work.

\section{OPEN MAPS: SHIFT AND INTERSECTION FAMILIES}

Open maps can be defined as the deterministic evolution corresponding to a given (closed) map followed by the loss of trajectories which pass through a given region in phase space. The set of trajectories that survives in the future and in the past defines the backward and forward trapped sets, respectively. In turn, the repeller is given by their intersection. The structure of this classical invariant can be very complex for chaotic dynamics, having a fractal dimension in most cases. Another relevant property of open maps is the decay rate, which is defined as the asymptotic loss of probability in one iteration.

In this work we study the shift and intersection families of open tribaker maps defined in [9] that differ only in their short-time dynamics, sharing the same repeller and decay rate. They are defined as preserving parity symmetry and, therefore, with the same openings in position and momentum.

In ternary notation the map action is given by a Bernoulli shift of $q=0 . \epsilon_{0} \epsilon_{1} \epsilon_{2} \ldots$ and $p=0 . \epsilon_{-1} \epsilon_{-2} \epsilon_{-3} \ldots$ (given by the corresponding trits $\left.\epsilon_{j}=0,1,2\right)$ as $(p \mid q)=$ $\ldots \epsilon_{-2} \epsilon_{-1} . \epsilon_{0} \epsilon_{1} \epsilon_{2} \ldots \rightarrow\left(p^{\prime} \mid q^{\prime}\right)=\ldots \epsilon_{-2} \epsilon_{-1} \epsilon_{0} . \epsilon_{1} \epsilon_{2} \ldots$,

where the dot is moved one position to the right. Shift and intersection families of open maps can be straightforwardly defined by replacing the usual trit $\epsilon_{j}$ with the open trit $\tilde{\epsilon}_{j}$, where the value 1 is forbidden (i.e., $\tilde{\epsilon}_{j}=0,2$ ). In this framework, the shift family members $\left(\mathcal{B}_{k}^{s}\right)$ are defined with two open trits corresponding to the $k$ th most significant trit of both position and momentum. Equivalently, the intersection family members $\left(\mathcal{B}_{k}^{i}\right)$ have the first $k$ trits open, in both position and momentum. The iterations of open baker maps in the unit square for $k=1,2$, and both, shift and intersection families are illustrated in Fig. 1. It is worth mentioning that the "cross"-shaped opening for $k=1$ is a direct consequence of having imposed the same projection in position and momentum.

The quantum version of the shift and intersection map families can be defined from the usual quantum tribaker map in a Hilbert space spanned by $l$ qutrits (with dimension $N=3^{l}$ )

$$
B_{\mathrm{pos}}=G_{N}^{\dagger} B_{\mathrm{mix}}=G_{N}^{\dagger}\left(\begin{array}{ccc}
G_{N / 3} & 0 & 0 \\
0 & G_{N / 3} & 0 \\
0 & 0 & G_{N / 3}
\end{array}\right),
$$

where the antiperiodic Fourier transform $G_{N}$ connects the position and momentum eigenvectors $\left(\left|q_{j}\right\rangle\right.$ and $\left|p_{j}\right\rangle$ with $j=1, \ldots, N)$,

$$
\left(G_{N}\right)_{j^{\prime}, j} \equiv\left\langle p_{j^{\prime}} \mid q_{j}\right\rangle=\frac{1}{\sqrt{N}} e^{-i \frac{2 \pi}{N}\left(j^{\prime}+\frac{1}{2}\right)\left(j+\frac{1}{2}\right)},
$$
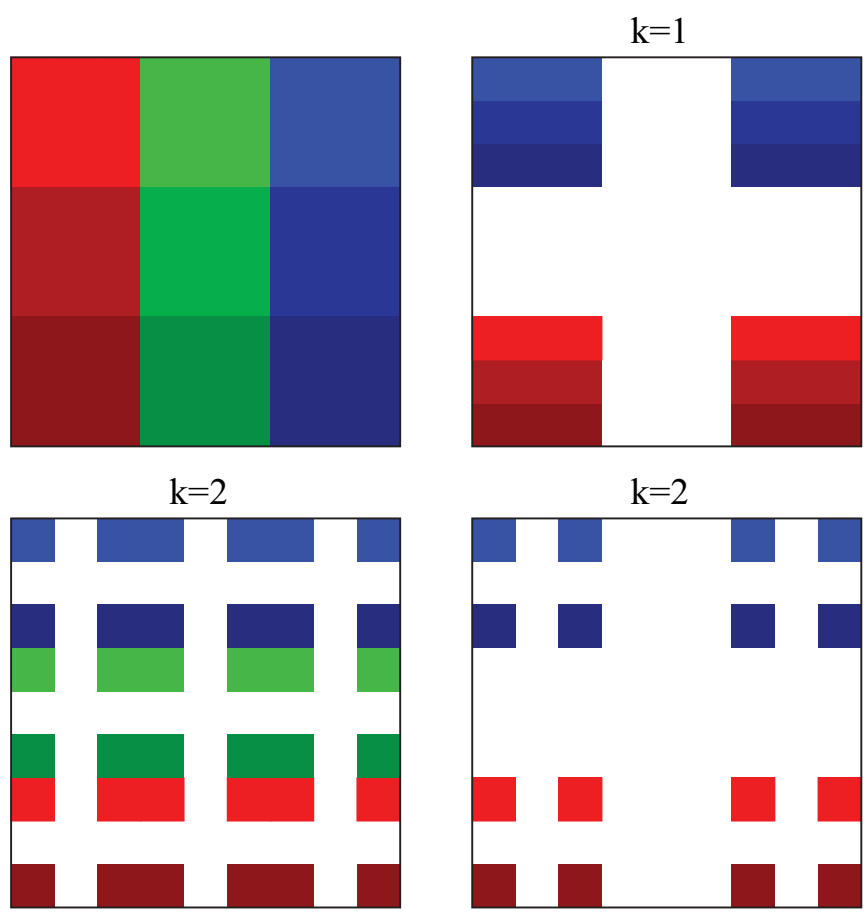

FIG. 1. (Color online) Iteration of the classical open baker map. The unit square divided into nine regions by means of three different gray intensities (three different colors) is shown at the top left. One iteration of the colored unit square with the open baker map is shown for $k=1$ (shift or intersection family) at the top right and for $k=2$ for the shift (bottom left) and intersection (bottom right) cases.

with $N=1 /(2 \pi \hbar)$ (see [10] and [11] for quantization details).

The forbidden 1 in one trit, $\tilde{\epsilon}$, can be quantized by means of the one-qutrit projector $\pi=I-|1\rangle\langle 1|=| 0\rangle\langle 0|+| 2\rangle\langle 2|$. In this way, the projector applied to the $i$ th qutrit can be written as

$$
\Pi_{i}=\underbrace{I \otimes \ldots \otimes I}_{i-1} \otimes \pi \otimes \underbrace{I \otimes \ldots \otimes I}_{l-i}
$$

with $\Pi_{i}=\Pi_{i}^{\dagger}, \Pi_{i}^{2}=\Pi_{i}$, and $\left[\Pi_{i}, \Pi_{j}\right]=0$ where $i, j=$ $1, \ldots, l$. Following this notation, the shift and intersection families of open quantum tribaker maps can be straightforwardly defined as

$$
\begin{gathered}
\tilde{B}_{k}^{s}=G_{N}^{\dagger} \Pi_{k} B_{\text {mix }} \Pi_{k}, \\
\tilde{B}_{k}^{i}=G_{N}^{\dagger} \Pi_{1}^{\dagger} \ldots \Pi_{k-1}^{\dagger} \Pi_{k}^{\dagger} B_{\text {mix }} \Pi_{k} \Pi_{k-1} \ldots \Pi_{1},
\end{gathered}
$$

with $k=1, \ldots, l$, and where the form $\tilde{B}_{\text {mix }}=\Pi B_{\text {mix }} \Pi$ preserves the parity symmetry of the closed map.

\section{TRANSIENT EFFECTS AND THE STATISTICS OF EIGENVALUES OF OPEN QUANTUM MAPS}

The geometry of phase space imposes restrictions in the asymptotic distributions of the eigenstates of quantum systems. In bounded systems of $f$ degrees of freedom, the well-known Weyl law establishes that the number of eigenstates $N(E)$ of energy less than $E$ is given by the number of cells of size $h^{f}$ contained in the corresponding allowed 
volume of phase space $V(E)$, i.e.,

$$
N(E)=V(E) /(2 \pi \hbar)^{f} .
$$

In open systems the relation between the geometry of the phase space and the statistics of eigenvalues is more involved. In these systems, the quantum evolution is given by a nonunitary operator with right and left decaying nonorthogonal eigenfunctions. The corresponding eigenvalues $z_{n}$ are complex numbers that fall inside the unit circle, that is, $\left|z_{n}\right|^{2} \leqslant 1$. The resonances are conjectured to be localized on invariant structures associated with the trapped sets; e.g., in the case of using the Husimi representation, right eigenmodes are localized on the unstable manifold of the trapped set [12-14]. This structure of the phase space has led to the prediction of a relation between the number of long-lived resonances and the fractal dimension $d$ of the repeller. As previously mentioned, the FWL establishes that the number of resonances with decay factor $\mu_{n}=\left|z_{n}\right|=\exp \left[-\Gamma_{n} / 2\right]>\mu$ scales as $N_{\mu} \sim N^{d / 2}$ in the semiclassical limit $N \rightarrow \infty$. This conjecture has been investigated in many systems, including quantum maps, twodimensional (2D) and three-dimensional (3D) billiards, and the modified Henon-Heiles potential $[3,5,6,15]$. Also, more scarring has been predicted in open systems than in closed ones [16,17]; this phenomenon has great consequences in many applications, such as microlasers [18].

The goal of this section is to test the validity of the FWL in the families of open tribaker maps introduced earlier. We remark that all these systems have the same repeller, classical information that determines the statistical behavior of the resonances which is embodied in the FWL. In Fig. 2 we show the ordered decay factor $\mu_{n}$ for the usual open tribaker map (i.e., the first member of any of the two families, $\tilde{B}_{1}^{s / i}$ ), having Hilbert space dimensions $N=3^{l}$ with $l=4,5,6,7,8$, and 9. In the inset in Fig. 2 we show the number of resonances with modulus $\mu_{n}>0.1$ as a function of $N$. Since the the fractal dimension of the repeller is $d=2 \ln (2) / \ln (3)$, it is clear from the inset that, for all the dimensions of the Hilbert space considered, $N_{\mu} \sim N^{d / 2}$ as predicted by the FWL.



FIG. 2. (Color online) Ordered decay factor $\mu_{n}$ of the usual open tribaker map as a function of $n / N$. Hilbert dimensions $N=3^{l}$ with $l=4$ [open (green) squares], $l=5$ [filled (blue) squares], $l=6$ [open (turquoise) circles], $l=7$ [filled (red) circles], $l=8$ [open (gray) triangles], and $l=9$ [filled (black) triangles]. Inset: Fraction of states $N_{\mu}$ with decay rate $\mu>0.1$ as a function of the Hilbert-space dimension. The solid (red) line corresponds to the prediction of the FWL $\left(N_{\mu} \sim N^{\ln (2) / \ln (3)}\right)$.

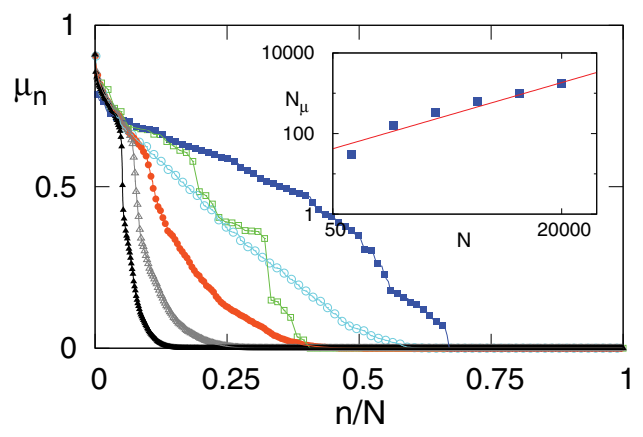

FIG. 3. (Color online) Ordered decay factor $\mu_{n}$ of the open map $\tilde{B}_{3}^{s}$ as a function of $n / N$. The Hilbert-space dimension is $N=3^{l}$ with $l=4$ [open (green) squares], $l=5$ [filled (blue) squares], $l=6$ [open (turquoise) circles], $l=7$ [filled (red) circles], $l=8$ [open (gray) triangles], and $l=9$ [filled (black) triangles]. Inset: Fraction of states $N_{\mu}$ with decay rate $\mu>0.1$ as a function of the Hilbert-space dimension. The solid (red) line corresponds to the prediction of the FWL $\left(N_{\mu} \sim N^{\ln (2) /(\ln (3))}\right)$.

In Figs. 3 and 4 we show the same statistics, but for the eigenvalues of the open maps $\tilde{B}_{3}^{s}$ and $\tilde{B}_{4}^{s}$, i.e., the third and fourth members of the shift family. For the open map $\tilde{B}_{3}^{s}$ (Fig. 3), only the distribution of resonances for the case of $N=3^{4}=81$ behaves differently than that of the other studied dimensions $N$. We note that the corresponding point deviates from the FWL as shown in the inset in Fig. 3. In the case of the open map $\tilde{B}_{4}^{s}$ (Fig. 4) the systematic imposed by the FWL fails for the distributions corresponding to $N=81,243$, and 729 . In order to see this assertion in more detail we compute the number of resonances with modulus $\mu_{n}>0.1$ as a function of $N$ for intermediate dimensions $N=81 \times i$ with $i=2,3,4 \ldots$ This is plotted by the dotted line in the inset in Fig. 4. We see that $N_{\mu}$ is a fluctuating function for $N \leqslant 3^{6}$; that for $N>3^{6}$ converges to the prediction of the FWL.

It is worth mentioning that we have also studied the other members of this family obtaining similar qualitative results as



FIG. 4. (Color online) Ordered decay factor $\mu_{n}$ of the open map $\tilde{B}_{4}^{s}$ as a function of $n / N$. The Hilbert-space dimension is $N=3^{l}$ with $l=4$ [open (green) squares], $l=5$ [filled (blue) squares], $l=6$ [open (turquoise) circles], $l=7$ [filled (red) circles], $l=8$ [open (gray) triangles], and $l=9$ [filled (black) triangles]. Inset: Fraction of states $N_{\mu}$ with decay rate $\mu>0.1$ as a function of the Hilbert-space dimension. The solid (red) line corresponds to the prediction of the FWL $\left(N_{\mu} \sim N^{\ln (2) /(\ln (3))}\right)$. Filled squares plot $N=3^{l}$ with $l=3,4$, $5,6,7,8$, and 9; dotted lines, $N=81 \times i$, with $i=2,3, \ldots$. 




FIG. 5. (Color online) Ordered decay factor $\mu_{n}$ of the open map $\tilde{B}_{2}^{i}$ as a function of $n / N$. The Hilbert-space dimension is $N=3^{l}$ with $l=4$ [open (green) squares], $l=5$ [filled (blue) squares], $l=6$ [open (turquoise) circles], $l=7$ [filled (red) circles], $l=8$ [open (gray) triangles], and $l=9$ [filled (black) triangles]. Inset: Fraction of states $N_{\mu}$ with decay rate $\mu>0.1$ as a function of the Hilbert-space dimension. The solid (red) line corresponds to the prediction of the $\operatorname{FWL}\left(N_{\mu} \sim N^{\ln (2) /(\ln (3))}\right)$.

those shown in Figs. 3 and 4, that is; after some $N$ the FWL starts to dictate the statistical behavior of the resonances.

Now we consider the statistics of the eigenvalues in the case of the intersection family. In Figs. 5 and 6, we show the statistics of the eigenvalues of maps $\tilde{B}_{2}^{i}$ and $\tilde{B}_{3}^{i}$, i.e., the second and third members of the intersection family. As for the examples that we have shown in the shift family, for the intersection maps the systematic imposed by the FWL is observed after some $N$. In the case of $\tilde{B}_{2}^{i}$ the FWL is observed after $N \sim 3^{6}$ and for $\tilde{B}_{3}^{i}$ the crossover is observed for $N \sim 3^{8}$ (see insets in Figs. 5 and 6).

Summarizing, the statistical behavior of the resonances of the open map of the shift and intersection families depends on $k$. For greater $k, N_{\mu}$ seems to follow the prediction of the FWL from a greater Hilbert dimension $N$. The reason for this behavior is as follows. As we can see in Fig. 1, the maps studied in this paper have smaller holes for greater $k$. When the size of the holes is comparable to $\hbar=1 / \sqrt{2 \pi N}$ it is expected that the semiclassical prediction of the FWL should not be valid due to

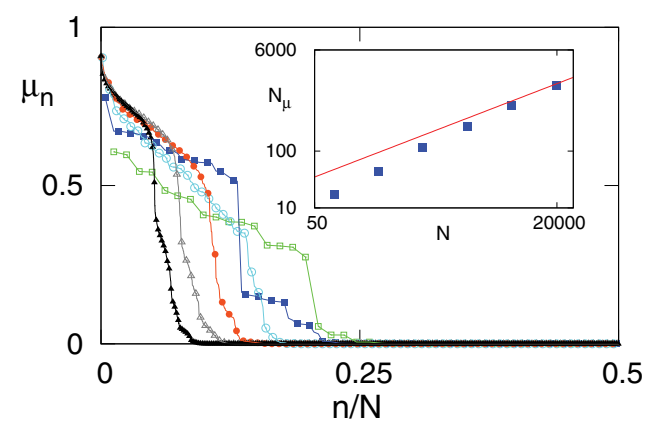

FIG. 6. (Color online) Ordered decay factor $\mu_{n}$ of the open map $\tilde{B}_{3}^{i}$ as a function of $n / N$. The Hilbert-space dimension is $N=3^{l}$ with $l=4$ [open (green) squares], $l=5$ [filled (blue) squares], $l=6$ [open (turquoise) circles], $l=7$ [filled (red) circles], $l=8$ [open (gray) triangles], and $l=9$ [filled (black) triangles]. Inset: Fraction of states $N_{\mu}$ with decay rate $\mu>0.1$ as a function of the Hilbert-space dimension. The solid (red) line corresponds to the prediction of the FWL $\left(N_{\mu} \sim N^{\ln (2) /(\ln (3))}\right)$. quantum effects such as diffraction (the exact source or sources for this are still being investigated). Following this reasoning, $N \sim 3^{2 k}$ seems to be a natural value for this transition to take place, but we were not able to precisely determine the value at which the FWL starts to rule the statistics of these maps. In the following section we go more deeply into the study of the classical support of open quantum maps.

\section{TRANSIENT EFFECTS AND THE THEORY OF SHORT PERIODIC ORBITS FOR OPEN QUANTUM MAPS}

The recently developed theory of short POs for open quantum maps [7,8] (which finds its roots in the corresponding theory for closed systems [19]) provides the ideal tool for testing to what point the repeller, more specifically the POs living in it, is the actual classical support of quantum resonances. The essential ingredient of this approach is the open scar function associated with each one of these trajectories.

Taking $\gamma$ as a PO of fundamental period $L$ that belongs to an open map, we define coherent states $\left|q_{j}, p_{j}\right\rangle$ associated with each point of the orbit (it has a total of $L$ points, all in the repeller) and construct the linear combination

$$
\left|\phi_{\gamma}^{m}\right\rangle=\frac{1}{\sqrt{L}} \sum_{j=0}^{L-1} \exp \left\{-2 \pi i\left(j A_{\gamma}^{m}-N \theta_{j}\right)\right\}\left|q_{j}, p_{j}\right\rangle .
$$

In this expression $m \in\{0, \ldots, L-1\}$ and $\theta_{j}=\sum_{l=0}^{j} S_{l}$, where $S_{l}$ is the action acquired by the $l$ th coherent state in one step of the map. The total action is $\theta_{L} \equiv S_{\gamma}$ and $A_{\gamma}^{m}=\left(N S_{\gamma}+m\right) / L$. The right and left scar functions for the PO are defined through the propagation of these linear combinations under the open map $\widetilde{U}$ (up to approximately the system's Ehrenfest time $\tau$ ):

$$
\left|\psi_{\gamma, m}^{R}\right\rangle=\frac{1}{\mathcal{N}_{\gamma}^{R}} \sum_{t=0}^{\tau} \widetilde{U}^{t} e^{-2 \pi i A_{\gamma}^{m} t} \cos \left(\frac{\pi t}{2 \tau}\right)\left|\phi_{\gamma}^{m}\right\rangle
$$

and

$$
\left\langle\psi_{\gamma, m}^{L}\right|=\frac{1}{\mathcal{N}_{\gamma}^{L}} \sum_{t=0}^{\tau}\left\langle\phi_{\gamma}^{m}\right| \widetilde{U}^{t} e^{-2 \pi i A_{\gamma}^{m} t} \cos \left(\frac{\pi t}{2 \tau}\right) .
$$

Normalization $\left(\mathcal{N}_{\gamma}^{R, L}\right)$ is chosen in such a way that $\left\langle\psi_{\gamma, m}^{R} \mid \psi_{\gamma, m}^{R}\right\rangle=\left\langle\psi_{\gamma, m}^{L} \mid \psi_{\gamma, m}^{L}\right\rangle$ and $\left\langle\psi_{\gamma, m}^{L} \mid \psi_{\gamma, m}^{R}\right\rangle=1$. We select a number of short POs that approximately cover the repeller, whose number scales following the FWL prediction [8]. Then we construct an appropriate basis in which we can write open evolution operators associated with open maps, i.e., we write the matrix $\left\langle\psi_{\alpha, i}^{L}|\widetilde{U}| \psi_{\beta, j}^{R}\right\rangle$, which in fact is an approximation to the open propagator $\widetilde{U}$ on the repeller. Given that $\left\langle\psi_{n}^{L} \mid \psi_{m}^{R}\right\rangle \neq \delta_{n m}$, we have to solve a generalized eigenvalue problem in order to obtain the eigenvectors of this matrix. This provides us with a classically motivated approximation to the system's long-lived resonances [7], which are constructed by the usual linear combination of the latter eigenvector's coefficients and the corresponding scar functions of the basis.

The short PO theory strongly relies on the repeller as the source of fundamental information in order to describe the long-lived portion of the spectra of open maps. In fact, it takes into account the details of the open dynamics on the 


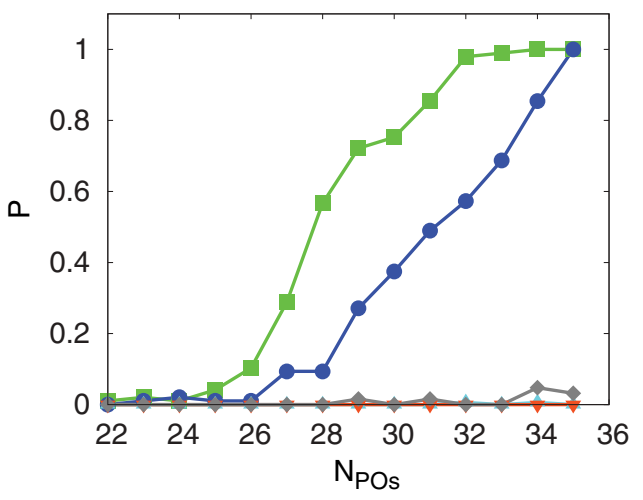

FIG. 7. (Color online) Performance $P$ of the short POs approach for the case of the shift family: fraction of long-lived resonances found as a function of the number of POs used in the calculations $N_{P O s}$. Hilbert space dimension is $N=3^{l}$ with $l=5$, all possible $k$ are shown. (Color) gray lines with squares correspond to $k=1$, circles to $k=2$, triangles up to $k=3$, triangles down to $k=4$, and diamonds to $k=5$.

repeller, going beyond the consideration of a measure such as, for instance, the fractal dimension which governs the FWL. But now the question arises: Is this enough, or are more elements needed?

We define the performance $P$ of this method as the fraction of long-lived eigenvalues that it is able to reproduce up to an error given by $\epsilon=$ $\sqrt{\left(\operatorname{Re}\left(z_{i}^{\mathrm{ex}}\right)-\operatorname{Re}\left(z_{i}^{\mathrm{PO}}\right)\right)^{2}+\left(\operatorname{Im}\left(z_{i}^{\mathrm{ex}}\right)-\operatorname{Im}\left(z_{i}^{\mathrm{PO}}\right)\right)^{2}}$, where $z_{i}^{\mathrm{ex}}$ and $z_{i}^{\mathrm{PO}}$ are the exact eigenvalues and those given by the short PO theory, respectively. In practice, we determine the number of exact eigenvalues with a modulus greater than 0.01 ; then we find for how many of them a semiclassical eigenvalue has been obtained in their $\epsilon=0.001$ vicinity; and, finally, we divide this number by the first one to obtain $P$. These threshold values guarantee the good performance of the approximation and the evaluation of a meaningful number of eigenvalues in the whole range of maps that we have studied. However, if detailed comparisons are needed, these values should be carefully adapted to each map, this going beyond the purposes of the present paper. In Figs. 7 and 8 we show $P$ as a function of the number of POs used, $N_{\text {POs }}$; here our method is applied to the shift and intersection families for $N=3^{5}$, respectively. At this relatively low value of $N$ it is already evident that the usual open tribaker map that corresponds to the $k=1$ value for both families proves to be well reproduced by this approximation. In the case of the shift family the good performance is suddenly lost when we arrive at the $k=l / 2$ threshold, i.e., from $k=3$ on, the method clearly fails. We note that the resonances of the shift family occupy classically forbidden regions for $k \geqslant l / 2$ [9]. Nevertheless, the performance is good for all members of the intersection family. We conjecture that this is because the probability outside the region roughly corresponding to the classical repeller is erased by means of the projector. Moreover, besides fluctuations probably connected with the different distributions of fixed points of the POs considered for the calculation and of the openings, it is clear that the performance is better for $k$ values $>1$ in the intersection family. We think that this is due to the same "erasing" effect that the projectors of this kind of map have. In other words, the finer holes in phase

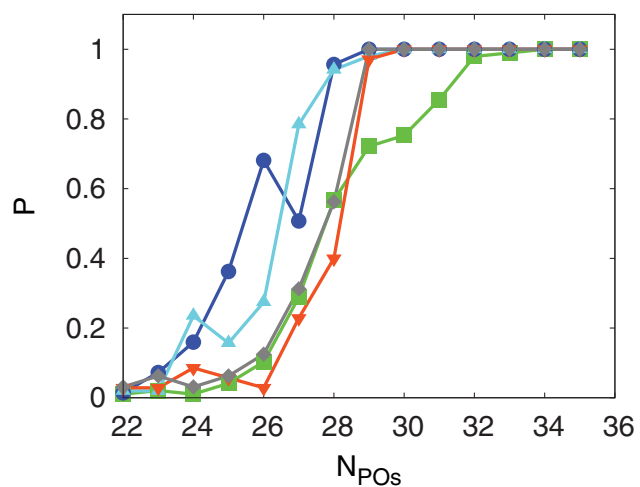

FIG. 8. (Color online) Performance $P$ of the short POs approach for the case of the intersection family: fraction of long-lived resonances found as a function of the number of POs used in the calculations $N_{P O s}$. Hilbert space dimension is $N=3^{l}$ with $l=5$, all possible $k$ are shown. We use the same colors and symbols as in Fig. 7.

space make resonances to occupy classically finer forbidden regions, which in turn are erased by the previous iteration of the openings. It is a sort of "fractal erasing effect" that constrains the probability to the classically allowed region. To further confirm this conjecture we have made the same calculation for the $l=6$ case, which qualitatively coincides with our previous reasoning. The corresponding results are shown in Figs. 9 and 10 .

In order to provide further insight on the differences among members of these two families of maps and relate them to basic features of general open (scattering) quantum systems, we study the phase-space distributions associated with the right and left eigenfunctions. For this purpose we use a recently introduced representation [20] that reveals the part of the quantum probability which is in the closest correspondence to the classical repeller. By defining the symmetrical operator

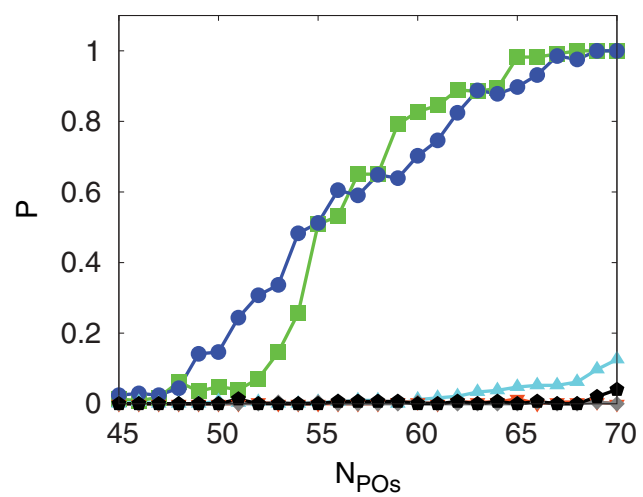

FIG. 9. (Color online) Performance $P$ of the short $P O$ approach in the case of the shift family: fraction of long-lived resonances found as a function of the number of POs used in the calculations $N_{\mathrm{POs}}$. The Hilbert-space dimension is $N=3^{l}$ with $l=6$; all possible $k$ values are shown. Line with squares corresponds to $k=1$; line with circles, to $k=2$; line with upward-pointing triangles, $k=3$; line with downward-pointing triangles, $k=4$; line with diamonds, to $k=5$; and line with pentagons, to $k=6$. 


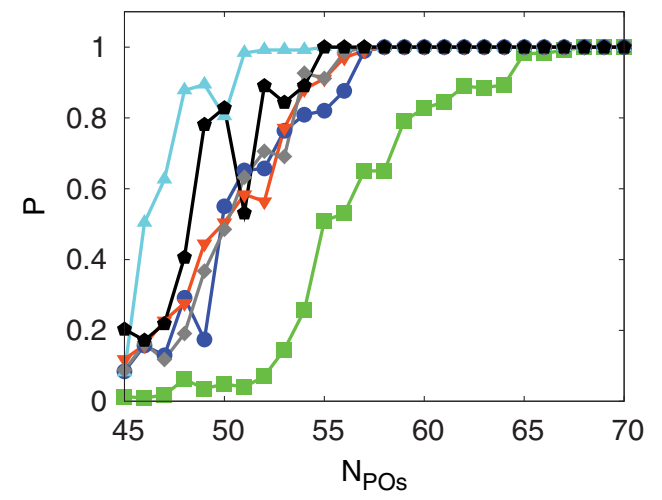

FIG. 10. (Color online) Performance $P$ of the short PO approach in the case of the intersection family: fraction of long-lived resonances found as a function of the number of POs used in the calculations $N_{\text {POs. }}$. The Hilbert-space dimension is $N=3^{l}$ with $l=6$; all possible $k$ values are shown. Colors and symbols are the same as in Fig. 9.

$\hat{h}_{j}$ associated with the right $\left|R_{j}\right\rangle$ and left $\left\langle L_{j}\right|$ eigenstates

$$
\hat{h}_{j}=\frac{\left|R_{j}\right\rangle\left\langle L_{j}\right|}{\left\langle L_{j} \mid R_{j}\right\rangle},
$$

which is related to the eigenvalue $z_{j}$, we construct the sum of the first $j$ of these projectors, ordered by decreasing modulus of the corresponding eigenvalues $\left(\left|z_{j}\right| \geqslant\left|z_{j^{\prime}}\right|\right.$ with $\left.j \leqslant j^{\prime}\right)$ :

$$
\hat{Q}_{j} \equiv \sum_{j^{\prime}=1}^{j} \hat{h}_{j^{\prime}} .
$$

The object to study consists of their phase-space representation by means of coherent states $|q, p\rangle$, which is given by

$$
\begin{gathered}
h_{j}(q, p)=\left|\left\langle q, p\left|\hat{h}_{j}\right| q, p\right\rangle\right|, \\
Q_{j}(q, p)=\left|\left\langle q, p\left|\hat{Q}_{j}\right| q, p\right\rangle\right| .
\end{gathered}
$$

In Fig. 11 we show $Q_{32}$ for the exact resonances and those given by the short PO approach, obtained for $l=5$ and $N_{\mathrm{POs}}=32$ (it is worth mentioning that we have observed similar results for $Q_{j}$ with $j$ around 32). In Figs. 11(a) and 11(b) the case $k=1$ (which coincides for both the shift and the intersection families) makes clear that the short PO theory is very accurate in reproducing not only the long-lived sector of the spectrum but also the component of the resonance eigenfunctions that live on the classical repeller. In fact, Figs. 11(a) and 11(b) are almost indistinguishable. This shows undoubtedly that the classical repeller is the meaningful support of long-lived states in this case. Although we do not show here the results for the intersection family, they all reproduce very well these components of the eigenstates, in agreement with the conclusions previously shown by means of the spectra. When the shift family is analyzed, again, a sort of phase transition behavior is observed, which spoils the short PO theory results in the same fashion as for the spectral values. This happens for $k \geqslant l / 2$; here $k=3$, the case which we show in Figs. 11(c) and 11(d). Moreover, in Figs. 11(e) and 11(f) we show the case $k=4$. It is important to analyze these results in detail. Though at first sight the exact $Q$ distribution for $k=3$ seems to be on the repeller, a closer look indicates that some probability escapes from the classically allowed region.
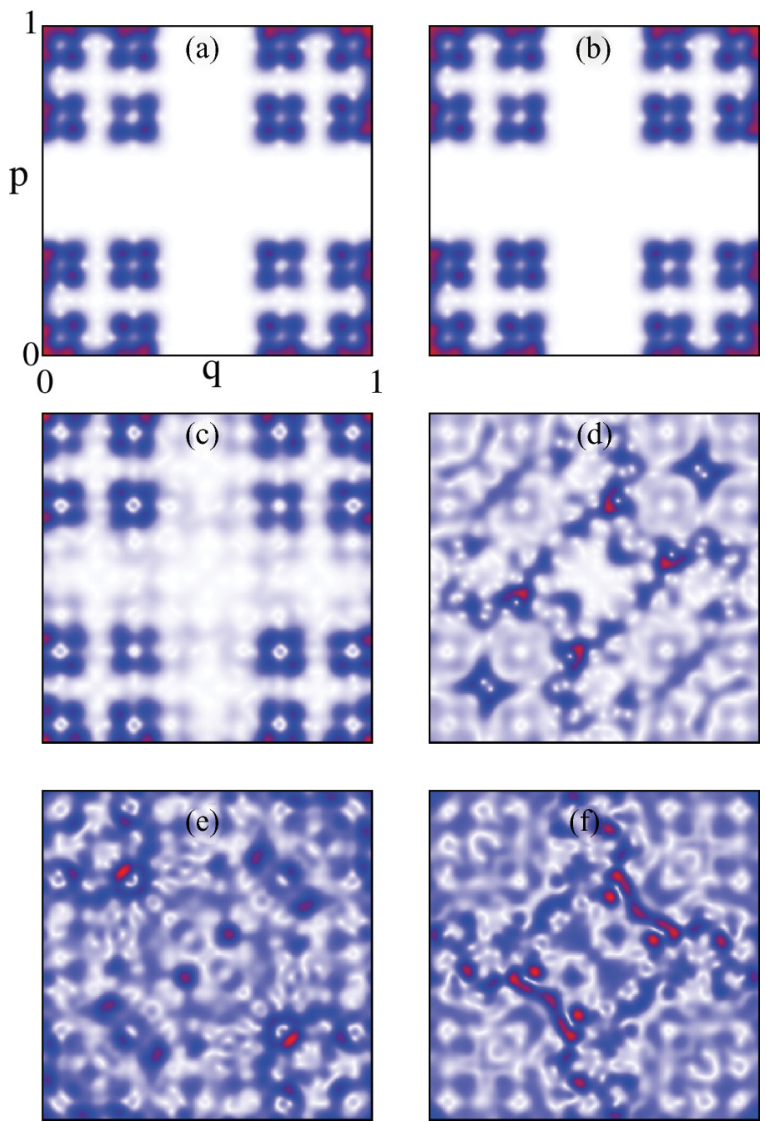

FIG. 11. (Color online) Phase-space representation of $Q_{32}$ for the exact and short PO approach with $l=5$ and $N_{\mathrm{POs}}=32$. A map with $k=1$ (both shift and intersection families) is shown (a) for exact eigenstates and (b) for the short PO approach. For cases of the shift family a map with $k=3,4$ shows (c, e) the exact eigenstates and $(\mathrm{d}, \mathrm{f})$ the short PO approach, respectively. The intensities of the phase-space representation are white for a 0 value and dark gray (red) for the maximum.

This is shown by the "ghost" images of the repeller, which can be clearly seen. Admittedly, the main contribution to $Q$ comes from the repeller, but these leaks are sufficient to spoil the performance of the semiclassical calculations, which can only faithfully reproduce the probability inside this invariant region, a fact that was recently verified in [8]. This is very important; it gives us another critical piece of information in order to construct a more powerful semiclassical theory for open maps. New ingredients in the shape of diffraction kernels and/or classical information outside of the repeller's area are needed. Identifying them will be the subject of future research. Finally, the results shown in Figs. 11(e) and 11(f) confirm that the semiclassical approach fails to describe the exact $Q$ when it clearly localizes outside of the classical repeller's area, as expected from a formulation that only takes into account the classical information inside of it.

\section{CONCLUSIONS}

At present, it is commonly accepted that the classical information contained in the repeller is the fundamental ingredient to describe quantum chaotic scattering systems. 
However, a recent study [9] has shown that transient features of the classical dynamics have crucial effects in the resonances of the quantum system.

In this paper we put this conclusion on firmer ground First, we have tested the validity of the FWL, one of the cornerstones of open chaotic quantum systems theory in a family of quantum open tribaker maps [9]. We have clearly shown that the statistics of the eigenvalues follows the FWL behavior from some dimension of the Hilbert space that depends on the $k$ of the map. In order to unveil the classical support of the resonances we have attempted to obtain them by means of the short PO approach. This theory was recently linked to the FWL in Ref. [8], and it is based on the short POs that live in the repeller. We have defined a measure of its performance, showing that it fails to describe the quantum resonances associated with $k \geqslant l / 2$ for the shift family of maps. In contrast, the intersection family, whose opening forces the probability to remain roughly inside what can be called the classical repeller's area, is very well reproduced. We provide some clues about the elements that would probably be needed in order to improve our semiclassical theory for open maps.

Summarizing, our results in open quantum maps have shown the need to include other ingredients, either classical and/or quantum, additionally to the repeller, in order to satisfactorily describe the quantum mechanics of scattering systems. Further investigations are needed in order to find them and extend the short PO theory for open quantum maps.

\section{ACKNOWLEDGMENTS}

This work was supported by the MICINN Spain under Contract No. MTM2009-14621, CEAL, and also CONICET, UBACyT, and ANPCyT (Argentina).
[1] U. Weiss, in Quantum Dissipative Systems (World Scientific, Singapore, 2008).

[2] S. Nonnenmacher, Nonlinearity 24, R123 (2011).

[3] W. T. Lu, S. Sridhar, and M. Zworski, Phys. Rev. Lett. 91, 154101 (2003).

[4] J. Sj ostrand, Duke Math. J. 60, 1 (1990); J. Sjöstrand and M. Zworski, ibid. 137, 381 (2007).

[5] J. A. Ramilowski, S. D. Prado, F. Borondo, and D. Farrelly, Phys. Rev. E 80, 055201(R) (2009); A. Eberspächer, J. Main, and G. Wunner, ibid. 82, 046201 (2010).

[6] H. Schomerus and J. Tworzydlo, Phys. Rev. Lett. 93, 154102 (2004); S. Nonnenmacher and M. Rubin, Nonlinearity 20, 1387 (2007); D. L. Shepelyansky, Phys. Rev. E 77, 015202(R) (2008).

[7] M. Novaes, J. M. Pedrosa, D. Wisniacki, G. G. Carlo, and J. P. Keating, Phys. Rev. E 80, 035202(R) (2009).

[8] J. M. Pedrosa, D. Wisniacki, G. G. Carlo, and M. Novaes, Phys. Rev. E 85, 036203 (2012).

[9] L. Ermann, G. G. Carlo, J. M. Pedrosa, and M. Saraceno, Phys. Rev. E 85, 066204 (2012).

[10] N. L. Balazs and A. Voros, Ann. Phys. 190, 1 (1989); M. Saraceno, ibid. 199, 37 (1990).

[11] L. Ermann and M. Saraceno, Phys. Rev. E 74, 046205 (2006).
[12] P. Gaspard, in Chaos, Scattering and Statistical Mechanics (Cambridge University Press, Cambridge, 1998).

[13] G. Casati, G. Maspero, and D. L. Shepelyansky, Physica D 131, 311 (1999).

[14] J. P. Keating, M. Novaes, S. D. Prado, and M. Sieber, Phys. Rev. Lett. 97, 150406 (2006).

[15] S. Nonnenmacher and M. Zworski, J. Phys. A 38, 10683 (2005).

[16] J. Wiersig, Phys. Rev. Lett. 97, 253901 (2006).

[17] D. A. Wisniacki and G. G. Carlo, Phys. Rev. E 77, 045201(R) (2008).

[18] W. Fang, A. Yamilov, and H. Cao, Phys. Rev. A 72, 023815 (2005); J. U. Nöckel and D. A. Stone, Nature (London) 385, 45 (1997); T. Harayama, P. Davis, and K. S. Ikeda, Phys. Rev. Lett. 90, 063901 (2003); J. Wiersig and M. Hentschel, Phys. Rev. A 73, 031802(R) (2006); Phys. Rev. Lett. 100, 033901 (2008).

[19] E. G. Vergini, J. Phys. A: Math. Gen. 33, 4709 (2000); E. G. Vergini and G. G. Carlo, ibid. 33, 4717 (2000); E. G. Vergini, D. Schneider, and A. F. Rivas, J. Phys. A: Math. Theor. 41, 405102 (2008); L. Ermann and M. Saraceno, Phys. Rev. E 78, 036221 (2008).

[20] L. Ermann, G. G. Carlo, and M. Saraceno, Phys. Rev. Lett. 103, 054102 (2009). 